\title{
Estudio y mejora de los avisos de aeródromo de helada y nieve
}

https://doi.org/10.31978/639-19-010-0.679

\author{
María Rosa Pons Reynés ${ }^{1}$ (mponsr@aemet.es) \\ Eroteida Sánchez García ${ }^{1}$ (esanchezg@aemet.es) \\ José Voces Aboy ${ }^{1}$ (jvocesa@aemet.es)
}

${ }^{1}$ AEMET / Delegación Territorial en Cantabria

\begin{abstract}
RESUMEN
El objetivo del presente estudio es analizar los avisos de aeródromo de helada y nieve de los aeropuertos de Vitoria y Pamplona desde la perspectiva del usuario: conocer su grado de satisfacción con los avisos emitidos y entender mejor las consecuencias que tienen en sus actividades la emisión de un aviso y cuestiones como la antelación con la que se emiten o los umbrales previstos.

El estudio ha permitido identificar una serie de mejoras en el procedimiento de elaboración de dichos avisos, las cuales está previsto que se incorporen a la normativa de AEMET.
\end{abstract}

PALABRAS CLAVE: helada; nieve; avisos; aeronáutica; usuarios; impacto.

\section{INTRODUCCIÓN}

Los avisos de aeródromo proporcionan información acerca de los fenómenos meteorológicos que podrían tener un efecto adverso en las aeronaves en tierra, incluidas las aeronaves estacionadas, y en las instalaciones y servicios del aeródromo. Son emitidos por las Oficinas Meteorológicas Principales de Aeródromo (OMPA) y distribuidos por la Oficina Meteorológica del Aeródromo (OMA) a todos los usuarios con los que existe un acuerdo: principalmente Aena, ENAIRE, compañías aéreas y los agentes de asistencia en tierra (handling).

Entre los fenómenos para los que se puede emitir un aviso de aeródromo se encuentran los relacionados con fenómenos invernales como son las heladas y la nieve. En aquellos aeropuertos que tienen riesgo de sufrir dichos fenómenos Aena pone en marcha al inicio de cada temporada el Plan de actuaciones invernales frente a contingencias de hielo y nieve, más conocido como Plan de invierno. En dicho plan se establecen las medidas que se deben tomar en los aeropuertos para minimizar el impacto de los citados fenómenos en las operaciones y garantizar la seguridad de las mismas, activándose la contingencia con la emisión de un aviso de aeródromo por parte de AEMET.

Si bien las incidencias debidas al hielo o la nieve en los aeropuertos de Aena representan un porcentaje muy pequeño sobre el total de las incidencias relacionadas con meteorología adversa (un 0,4\% en 2017 según datos de Aena), son de las más costosas. La campaña 2017-2018 contó con un presupuesto de 2,3 millones de euros, disponiéndose de más de 100 vehículos para la retirada de nieve y hielo, más de 450 toneladas de fundentes e impartiendo formación específica a casi 900 trabajadores.

Por ello, se ha considerado importante hacer un estudio del estado actual de los avisos de helada y nieve, analizando especialmente las necesidades de los usuarios. Si bien se ha realizado también una verificación 
objetiva de los avisos emitidos durante los últimos cinco años, los objetivos principales del trabajo son conocer el grado de satisfacción de los usuarios con los avisos emitidos de heladas y nieve, y entender mejor las consecuencias que tienen en sus actividades dichos avisos y cuestiones como la antelación con la que se emiten o los umbrales previstos.

El trabajo se ha estructurado incluyendo en primer lugar una breve climatología de los fenómenos de helada y nieve, y a continuación una descripción de la información que contienen los avisos. En el apartado 4 se describen las acciones llevadas a cabo con los usuarios, en el quinto se realiza la verificación y análisis de los avisos emitidos y en último lugar se describen las principales conclusiones del trabajo.

El estudio se ha centrado en los aeropuertos de Vitoria (LEVT) y Pamplona (LEPP), los dos aeropuertos bajo el área de responsabilidad de la OMPA de Santander en los que los fenómenos indicados son más relevantes.

\section{CLIMATOLOGÍA DE HELADAS Y NIEVE}

Los aeropuertos de Vitoria y Pamplona son el tercer y cuarto aeropuerto de la red de Aena respectivamente con mayor número de días de nieve al año, solo superados por Burgos y León. En la tabla 1 se recogen los valores climatológicos de los días de helada y nieve en el periodo 1981-2010 (AEMET, 2012), en la que queda de manifiesto la gran variabilidad interanual que presentan dichas variables.

\begin{tabular}{|l|c|c|}
\cline { 2 - 3 } \multicolumn{1}{c|}{} & LEPP & LEVT \\
\hline Altitud & $513 \mathrm{~m}$ & $459 \mathrm{~m}$ \\
\hline № medio días nieve/año & 10 & 11 \\
\hline № mínimo-máximo días nieve/año & $1-24$ & $0-32$ \\
\hline № medio días helada/año & 39 & 49 \\
\hline № mínimo-máximo días helada/año & $11-64$ & $24-72$ \\
\hline
\end{tabular}

Tabla 1. Altitudes y valores climatológicos de días de nieve y helada en el periodo 1981-2010.

\section{CONTENIDO DE LOS AVISOS DE AERÓDROMO}

La normativa interna de AEMET sobre los avisos de aeródromo viene recogida en la Guía de elaboración de avisos de aeródromo (MPO-GUI-0302), siguiendo las directrices establecidas en el Anexo 3 de la OACI (OACI, 2016). Los avisos se pueden emitir con una antelación máxima de 24 horas, y su periodo de validez puede abarcar como máximo 24 horas, debiéndose emitir uno nuevo antes de que finalice dicho periodo si se prevé que la duración será mayor.

Además de especificar la duración del aviso, los avisos de nieve y helada permiten especificar de forma opcional el espesor de nieve y la temperatura mínima respectivamente, con un formato muy sencillo. Se incluyen a continuación un par de ejemplos de avisos, de $3 \mathrm{~cm}$ de espesor de nieve y de una helada con una temperatura mínima prevista de $-4{ }^{\circ} \mathrm{C}$ :

\section{LEVT AD WRNG 1 VALID 192000/201000 SN 03CM FCST NC= LEPP AD WRNG 1 VALID 222000/230800 FROST T MS04C FCST NC=}

Los avisos de helada pueden llevar un calificador de intensidad moderada (MOD, para temperaturas entre $-4{ }^{\circ} \mathrm{C}$ y $-10^{\circ} \mathrm{C}$ ) o fuerte (HVY, para temperaturas inferiores a $-10^{\circ} \mathrm{C}$ ), y los de nieve solo de HVY. Para la emisión de una actualización se requiere una variación superior a $5 \mathrm{~cm}$ de espesor de nieve o superior a $2{ }^{\circ} \mathrm{C}$ en la temperatura mínima (siempre que se mantenga por debajo de $0{ }^{\circ} \mathrm{C}$ ). 


\section{ACCIONES CON LOS USUARIOS}

Con el fin de conocer el grado de satisfacción de los usuarios con los avisos de helada y nieve y los detalles de las medidas incluidas en su plan de invierno se les remitió una encuesta de valoración y se celebró una reunión con ellos en cada aeropuerto. En los siguientes apartados se presentan los resultados de dichas encuestas y un resumen del contenido de sus planes de invierno, junto con las sugerencias planteadas por los usuarios en las reuniones celebradas.

\subsection{Encuestas de valoración}

La encuesta fue rellenada por los usuarios antes de las reuniones y de forma anónima, identificándose únicamente el tipo de usuario (gestor, compañía aérea, etc.).

Se recibieron seis encuestas de valoración en cada aeropuerto: en Pamplona, 4 de Operaciones de Aena, 1 de la Torre de Control y 1 de una empresa de handling; en Vitoria, 2 de Operaciones de Aena, 1 de la Torre de Control y 3 de empresas de handling. En la tabla 2 se muestra la media de las puntuaciones otorgadas a la utilidad, la antelación y la confianza de los avisos, sobre un máximo de 10.

\begin{tabular}{|l|c|c|c|c|}
\cline { 2 - 5 } \multicolumn{1}{c|}{} & \multicolumn{2}{c|}{ HELADA } & \multicolumn{2}{c|}{ NIEVE } \\
\cline { 2 - 5 } \multicolumn{1}{c|}{} & LEPP & LEVT & LEPP & LEVT \\
\hline Utilidad & 8,7 & 8,5 & 9 & 8,7 \\
\hline Antelación & 9,2 & 7,7 & 9,2 & 7,8 \\
\hline Confianza & 8,8 & 8,3 & 8,8 & 8,7 \\
\hline
\end{tabular}

Tabla 2. Valoración de los usuarios sobre los avisos de helada y nieve.

Las valoraciones recibidas fueron muy altas, recibiendo únicamente una puntuación inferior a 8 la antelación de los avisos en Vitoria. Así mismo, todos los usuarios consideraron que el número de falsas alarmas y de eventos no previstos era razonable.

En relación con los valores previstos, la temperatura mínima del aviso se considera en general bien prevista en ambos aeropuertos, así como el espesor observado de nieve en Pamplona. En Vitoria no obstante, el 50 \% de los usuarios considera que el espesor observado es algo inferior al previsto.

En cuanto a la antelación adecuada y límite que consideran los usuarios para los avisos, hubo resultados muy dispares en las encuestas, incluso entre las recibidas del mismo colectivo. En este sentido fue más esclarecedor tratar el tema directamente con los usuarios en la reunión (véase el apartado siguiente).

\subsection{Reuniones con los usuarios. Planes de invierno y necesidades}

Se celebró una reunión en cada aeropuerto el 22 de marzo de 2018, a la que asistieron representantes de AEMET, Aena, ENAIRE y compañías de asistencia en tierra. En ella se revisaron las actuaciones principales de los planes de invierno, con el fin de identificar las cuestiones más críticas para los usuarios.

Al recibir un aviso de nieve o helada, Aena toma una serie de medidas preventivas. Por un lado, organizando los recursos humanos necesarios, con prolongaciones de horario, adelantando inicios de turno y/o reforzando plantillas. Por otro lado, preparando los equipos y vehículos de deshielo. Solo en algunas condiciones de suelo mojado, se realiza un tratamiento preventivo sobre las pistas aplicando fundentes aproximadamente una hora antes (en suelo seco no resulta eficiente). La aplicación preventiva es delicada ya que el fundente solo actúa durante un tiempo limitado; si el episodio se retrasa dicha prevención habrá sido en balde y se requerirá una nueva.

Una vez iniciado el episodio, se realizan tratamientos con fundentes sobre el suelo ya húmedo y se deshielan las aeronaves. Así mismo, vehículos especiales realizan medidas continuas del coeficiente de fricción y del espesor de la nieve, retirándose la nieve de la pista una vez que el espesor sea superior a 1,5 cm aproximadamente. Con espesores superiores a $5 \mathrm{~cm}$ con nieve seca (e inferiores con nieve húmeda) o coeficientes de 
fricción inferiores a 0,2 aproximadamente, el aeropuerto queda cerrado al tráfico hasta que mejoren las condiciones. Además, si se prevé que la duración del cierre será superior a tres horas, el aeropuerto debe emitir un aviso especial (NOTAM).

Se identificaron, por tanto, una serie de cuestiones críticas en las que los usuarios plantearon sus necesidades:

- La importancia de que los avisos incluyan siempre el espesor de nieve o la temperatura mínima prevista (especialmente el primero), de forma que ellos puedan dimensionar mejor los recursos necesarios.

- Poder disponer de los avisos con la suficiente antelación para tomar las medidas oportunas. Esto incluye las renovaciones, sobre las que los usuarios indicaron que se recibían con muy poco margen. La antelación que se considera límite es diferente para Vitoria, que está operativo las 24 horas y Pamplona, que permanece cerrado por la noche. El primero planteó un mínimo de 6 horas antes del inicio del episodio y el segundo un mínimo de 2 horas antes del cierre.

- Acotar lo mejor posible el inicio y fin del episodio. Este aspecto era más crítico para LEPP que, al no estar operativo las 24 horas, ante un aviso previsto durante el horario de cierre tenía que decidir si prolongaban su jornada los trabajadores de ese mismo día o adelantaban su entrada los del día siguiente.

\section{ANÁLISIS DE LOS AVISOS EMITIDOS}

En el presente estudio se han analizado los avisos de helada y nieve emitidos en LEVT y LEPP durante algo más de 5 años (del 1 de enero de 2013 al 28 de febrero de 2018): 263 avisos de helada en LEPP y 354 en LEVT, así como 56 avisos de nieve en LEPP y 73 en LEVT (incluyendo los cancelados). Como datos de observación se han utilizado los datos de las estaciones climatológicas 9263D (LEPP) y 9091O (LEVT), así como los informes METAR, dependiendo de la variable y el aeropuerto:

- Para la temperatura mínima: datos horarios de las estaciones 9263D y 90910.

- Para la nieve: datos diarios de ocurrencia de nieve en 9263D y 9091O. En LEVT y por ser H24, también informes METAR.

En el caso de la nieve, es importante señalar que ante la falta de datos de observación de espesores, solo se ha podido verificar su ocurrencia.

\subsection{Ocurrencia de heladas y nieve}

Considerando solo los años completos (2013-2017), el número de avisos emitidos implica una media de 46 avisos de helada/año y 10 avisos de nieve/año en LEPP, y 65 avisos de helada/año y 12 avisos de nieve/año en LEVT. No obstante, tal y como cabe esperar, la variabilidad interanual de los avisos emitidos es muy grande como se puede ver en la figura 1.

La duración de los avisos es también muy variable, especialmente los de nieve. Se ha podido verificar la duración de las heladas previstas frente a las observadas, obteniéndose de media 8,5 horas para las heladas previstas tanto en LEPP y LEVT. La duración media de las heladas observadas ha sido muy similar, 7 horas en LEPP y 8 horas en LEVT. Se comprueba también que la hora de finalización más común de las heladas observadas son las 8 UTC.

En la duración de los avisos de nieve, llama la atención el número tan elevado de avisos que abarca el máximo permitido de 24 horas, especialmente en el caso de LEVT. Este hecho, además de dar una indicación de que los episodios de nieve tienen una duración considerable, tiene su importancia cuando se aborde el tema de la antelación de los avisos, en el apartado 4.3. 

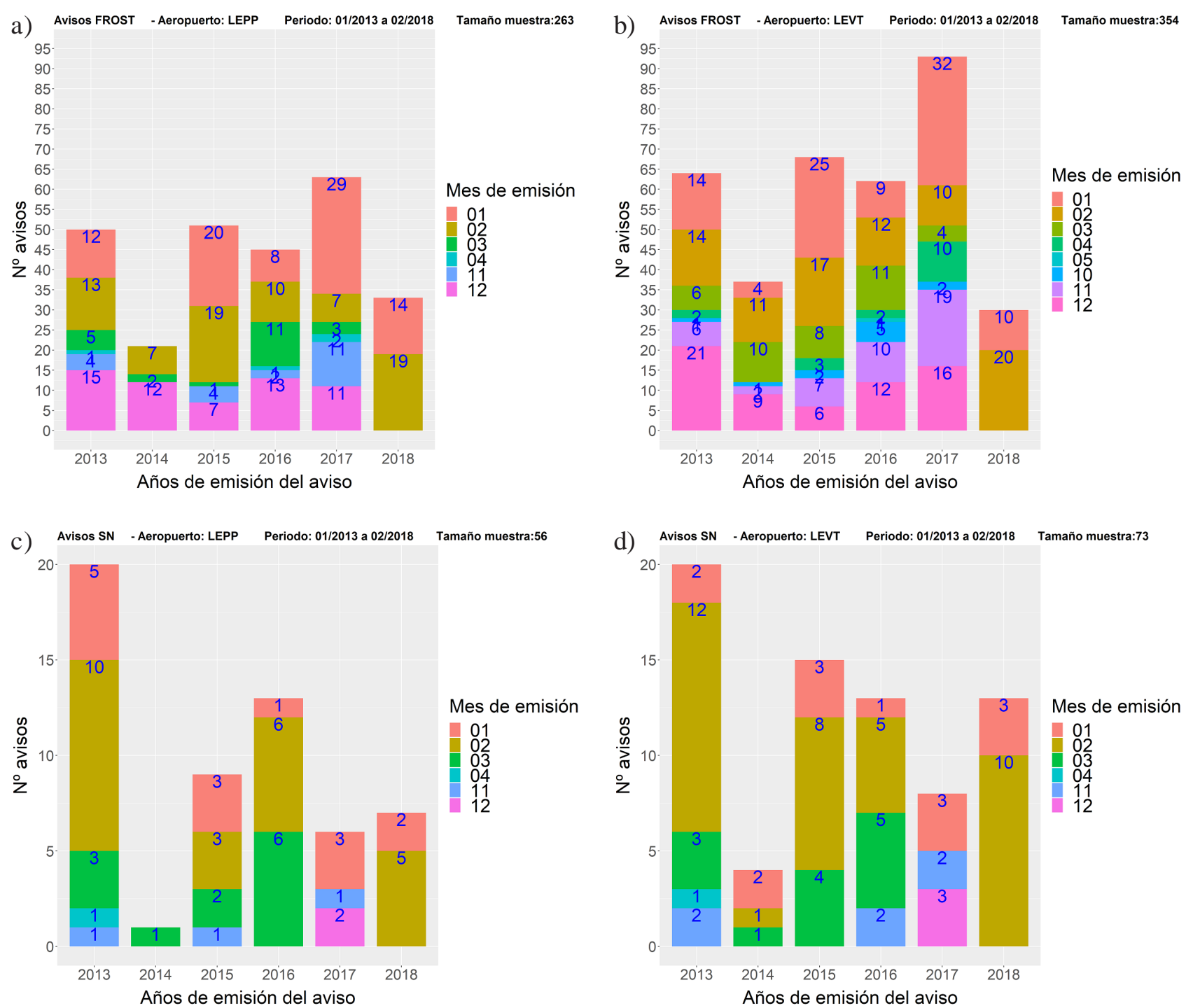

Figura 1. Avisos de helada y nieve emitidos por meses y años en el periodo del 1 de enero de 2013 al 28 de febrero de 2018. De izquierda a derecha y de arriba abajo: a) avisos de helada en LEPP,

b) avisos de helada en LEVT, c) avisos de nieve en LEPP y d) avisos de nieve en LEVT.

Se han calculado los índices POD (Probability of Detection, proporción de los eventos observados que fueron previstos) y FAR (False Alarm Ratio, proporción de los eventos previstos que no fueron observados), recogiéndose sus valores en la tabla 3. Las heladas se han verificado con las observaciones horarias de la estación climatológica y las nevadas de forma diferente según el aeropuerto: en LEPP, con la ocurrencia diaria de nieve de la estación climatológica y en LEVT, con los informes METAR.

\begin{tabular}{|c|c|c|c|c|}
\cline { 2 - 5 } \multicolumn{1}{c|}{} & \multicolumn{2}{c|}{ POD } & \multicolumn{2}{c|}{ FAR } \\
\cline { 2 - 5 } \multicolumn{1}{c|}{} & LEPP & LEVT & LEPP & LEVT \\
\hline Helada & $91 \%$ & $97 \%$ & $33 \%(13 \%)$ & $42 \%(19 \%)$ \\
\hline Nieve & $79 \%$ & $80 \%(89 \%)$ & $16 \%$ & $16 \%$ \\
\hline
\end{tabular}

Tabla 3. Probabilidad de detección (POD) y falsas alarmas (FAR) en porcentaje. Los valores entre paréntesis se obtienen siendo más permisivo con lo que se considera un acierto. En el caso de las heladas, no se consideran falsas alarmas valores de temperatura entre 0 y $1^{\circ} \mathrm{C}$ y en el caso de la nieve, no se considera un evento no previsto si solo se ha cifrado nieve débil en uno o dos METAR. 


\subsection{Valores previstos de temperatura mínima y espesor}

En el apartado 3 se indicó que, con la normativa actual, la inclusión de los valores previstos de temperatura mínima y espesor no es obligatoria. En los avisos emitidos en el periodo de estudio, se ha podido constatar que la proporción de avisos en los que se especifican dichos valores es baja: el $18 \%$ de los avisos de helada de LEPP y el 16\% de los avisos de LEVT especifican la temperatura mínima; el $31 \%$ de los avisos de nieve de LEPP y el $45 \%$ de los avisos de LEVT especifican el espesor de nieve previsto.

No obstante, en relación con los avisos de helada se ha comprobado que cuando se ha tratado de heladas moderadas (no se han observado heladas fuertes), la mayoría de los avisos especificaban la temperatura mínima.

En la verificación realizada de los valores de temperatura mínima previstos (figura 2), se ha visto que en casi el $80 \%$ de los casos la temperatura mínima observada difería en menos de $2{ }^{\circ} \mathrm{C}$ de la prevista.
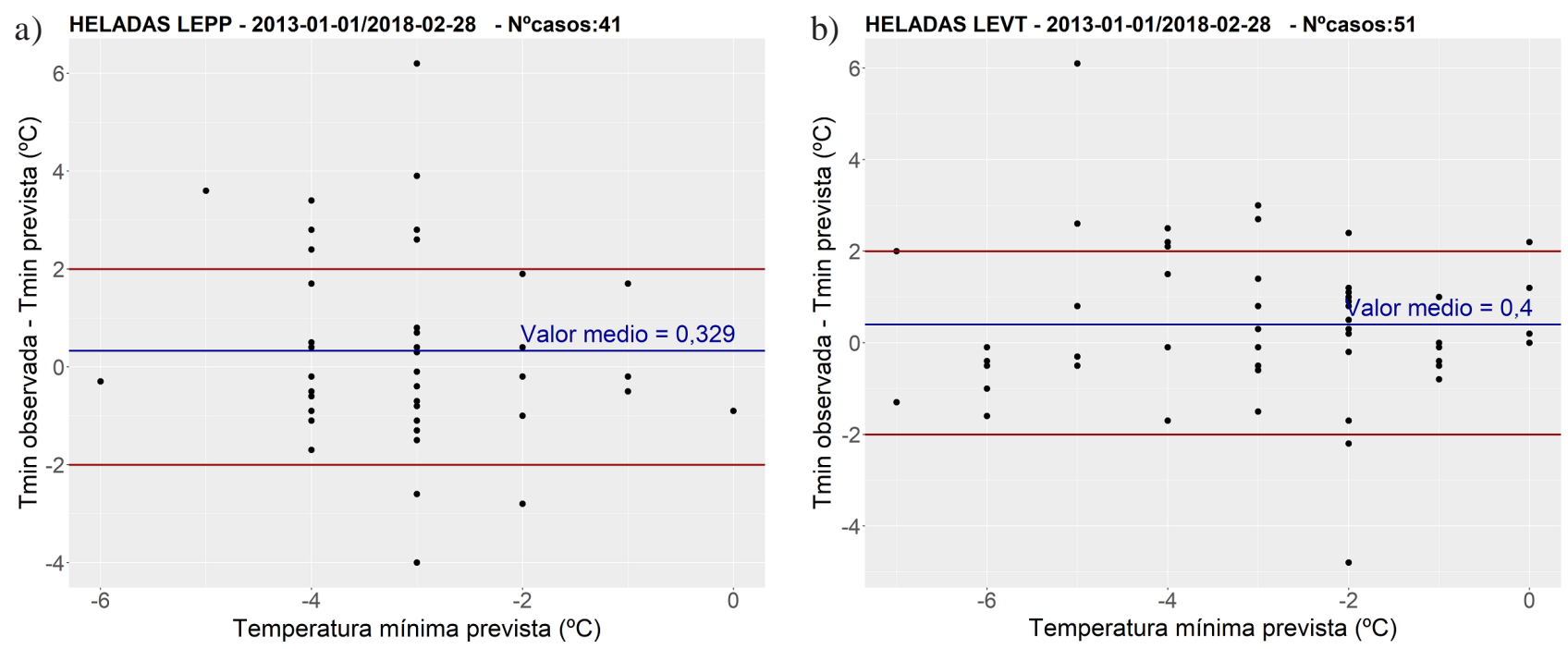

Figura 2. Temperatura mínima observada menos temperatura mínima prevista, frente a temperatura mínima prevista en: a) LEPP y b) LEVT. Los puntos entre las dos líneas rojas suponen una diferencia inferior a $2^{\circ} \mathrm{C}$ entre el valor previsto y el observado.

\subsection{Antelación y hora de emisión de los avisos}

En el caso de las heladas, se ha comprobado que la mayor parte de los avisos se han emitido entre las $15 \mathrm{y}$ las 20 UTC del día anterior. No obstante, en varios episodios en LEPP no se han emitido con las dos horas antes del cierre del aeropuerto que el usuario considera necesarias.

Por último, se ha analizado la antelación con la que se emiten los avisos, en función del número de horas transcurridas desde la emisión del aviso hasta que se prevé el inicio del episodio. Se ha podido constatar que en un número excesivamente elevado de casos, la antelación es de 0 horas, coincidiendo la hora de emisión del aviso con el inicio del periodo de validez del episodio.

En el caso de los avisos de nieve se ha podido comprobar que gran parte de los casos de antelación 0 horas se corresponden con las renovaciones de avisos que duran 24 horas. Es decir, se emite un aviso de nieve de 24 horas de duración y durante la última hora de su periodo de validez, se emite uno nuevo prolongando el episodio unas horas más. El predictor responsable del aviso considera en general que el usuario ya está sobre aviso y que además cuenta con toda la información de la duración de nieve prevista en el informe TAF. Sin embargo, esta percepción debe corregirse porque los destinatarios de los productos son diferentes y son los avisos de aeródromo los que desencadenan las acciones incluidas en los planes de invierno y no los informes TAF. 


\section{CONCLUSIONES}

Se ha podido comprobar que el grado de satisfacción de los usuarios de los aeropuertos de Vitoria y Pamplona con los avisos de helada y nieve en general es alto, y que los índices de probabilidad de detección (entre el $79 \%$ y el $97 \%$ ) y las falsas alarmas (entre el $13 \%$ y el 19\%) son buenos. Así mismo, el $80 \%$ de las temperaturas mínimas previstas difieren en menos de $2{ }^{\circ} \mathrm{C}$ de las observadas.

No obstante, se han identificado una serie de mejoras que se deberían implementar para atender mejor las necesidades de los usuarios: especificar siempre el valor de la temperatura mínima y el espesor previsto, emitir los avisos y las renovaciones con un mínimo de 6 horas de antelación en el caso de Vitoria y al menos dos horas antes del cierre en el caso de Pamplona, y ajustar bien las horas de inicio y fin del episodio.

Si bien estas conclusiones se han obtenido para Vitoria y Pamplona, son fácilmente extrapolables al resto de aeródromos, con ligeros cambios en las antelaciones necesarias. Por ello, AEMET tiene previsto modificar su normativa interna sobre la elaboración de avisos (MPO-GUI-0302) de cara a la temporada invernal 2018-2019: fijar unos mínimos para la antelación de los avisos y especificar siempre la temperatura mínima y el espesor de nieve previsto.

En estas circunstancias, sería deseable promover las observaciones de espesor de nieve de forma sistemática en las OMA y así poder verificar mejor los espesores previstos.

Los usuarios han valorado muy positivamente la iniciativa de AEMET de revisar los avisos de helada y nieve; el estudio ha permitido introducir una serie de mejoras en el proceso de elaboración de dichos avisos, atendiendo a sus necesidades. Como trabajo futuro sería aconsejable revisar los avisos de aeródromo correspondientes a otros fenómenos meteorológicos, y poder identificar así nuevas oportunidades de mejora.

\section{AGRADECIMIENTOS}

Los autores desean agradecer la colaboración de los usuarios de los aeropuertos de Vitoria y Pamplona (Aena, ENAIRE y empresas de asistencia en tierra) por rellenar las encuestas y aportar sugerencias, y de Inmaculada Benito Peraita (jefa de la OMA de Vitoria) y Pedro Oria Iriarte (delegado territorial de AEMET en Navarra) por las gestiones realizadas con dichos usuarios.

\section{REFERENCIAS}

AEMET, 2010. MPO-GUI-0302. Guía de elaboración de los avisos de aeródromo.

AEMET, 2012. Guía resumida del clima en España (1981-2010). Versión 3.0. NIPO: 281-12-01.

OACI, 2016. Anexo 3 al Convenio sobre Aviación Civil Internacional. Servicio Meteorológico para la navegación aérea internacional. XIX edición. 
